\title{
On the regularization of solution of an inverse ultraparabolic equation associated with perturbed final data
}

Nguyen Huy Tuan', Vo Anh Khoa ${ }^{2,3 *}$, Le Trong Lan ${ }^{3}$ and Tran The Hung ${ }^{3}$

\section{"Correspondence:}

khoa.vo@gssi.infn.it

${ }^{2}$ Mathematics and Computer

Science Division, Gran Sasso Science

Institute, Viale Francesco Crispi 7,

L?Aquila, Abruzzo 67100, Italy

${ }^{3}$ Department of Mathematics and

Computer Science, Ho Chi Minh

City University of Science, 227

Nguyen Van Cu Street, District 5, Ho

Chi Minh City, Vietnam

Full list of author information is

available at the end of the article

\begin{abstract}
In this paper, we study the inverse problem for a class of abstract ultraparabolic equations which is well known to be ill-posed. We employ some elementary results of semi-group theory to present the formula of solution, then show the instability cause. Since the solution exhibits unstable dependence on the given data functions, we propose a regularization method to stabilize the solution, then obtain the error estimate. A numerical example shows that the method is efficient and feasible. This work slightly extends the earlier results in Zouyed and Rebbani (J. Inverse III-Posed Probl. 22(4):449-466, 2014).
\end{abstract}

MSC: 47A52; 20M17; 26D15

Keywords: ultraparabolic equation; ill-posed problem; semi-group method; stability; error estimate

\section{Introduction}

Let us denote by $\|\cdot\|$ the norm and by $\langle\cdot, \cdot\rangle$ the inner product in $L^{2}(0, \pi)$, i.e.,

$$
\langle u, v\rangle=\int_{0}^{\pi} u v d x, \quad\|u\|=\sqrt{\int_{0}^{\pi}|u|^{2} d x} .
$$

In this paper, we consider the following problem: determine a function $u:[0, T] \times$ $[0, T] \rightarrow L^{2}(0, \pi)$ solution to the Cauchy problem

$$
\begin{cases}u_{t}+u_{s}-\Delta u=f(x, t, s), & (x, t, s) \in[0, \pi] \times[0, T] \times[0, T], \\ u(0, t, s)=u(\pi, t, s)=0, & (t, s) \in[0, T] \times[0, T], \\ u(x, T, s)=\psi(x, s), & (x, s) \in[0, \pi] \times[0, T], \\ u(x, t, T)=\varphi(x, t), & (x, t) \in[0, \pi] \times[0, T],\end{cases}
$$

with corresponding perturbed data functions $\left(\psi^{\varepsilon}, \varphi^{\varepsilon}\right)$ satisfying

$$
\left\|\psi^{\varepsilon}-\psi\right\| \leq \varepsilon, \quad\left\|\varphi^{\varepsilon}-\varphi\right\| \leq \varepsilon,
$$

() 2015 Tuan et al.; licensee Springer. This is an Open Access article distributed under the terms of the Creative Commons Attribution License (http://creativecommons.org/licenses/by/4.0), which permits unrestricted use, distribution, and reproduction in any medium, provided the original work is properly credited. 
where $\psi^{\varepsilon}$ and $\varphi^{\varepsilon}$ play roles as perturbed functions and $\varepsilon>0$ represents a bound between the exact function $(\varphi, \psi)$ and the perturbed one $\left(\varphi^{\varepsilon}, \psi^{\varepsilon}\right)$ over $L^{2}(0, \pi)$ and the given function $f$ is called the source function.

Ultraparabolic equations arise in several areas of science, such as mathematical biology in population dynamics [1] and probability in connection with multi-parameter Brownian motion [2], and in the theory of boundary layers [3]. From those applications, ultraparabolic equations have gained considerable attention in many mathematical aspects (see, e.g., $[1,4-9]$ and the references therein).

In the mathematical literature, various types of ultraparabolic problems have been solved. There have been some papers dealing with the existence and uniqueness of solution for many kinds of ultraparabolic equations, e.g., $[1,10,11]$. As the pioneer in numerical methods for such equations, Akrivis et al. [5] numerically approximated the solution of a prototype ultraparabolic equation by applying a fixed-step backward Euler scheme and a second-order box-type finite difference method. Some extension works for the numerical angle that should be mentioned are $[12,13]$ by Ashyralyev and Yilmaz, and Marcozzi, respectively. We also remark that, in general, ultraparabolic equations do not possess properties that are closely fundamental to many kinds of parabolic equations including strong maximum principles, a priori estimates, and so on.

In the phase of ill-posed ultraparabolic problems, the authors Zouyed and Rebbani very recently proposed in [7] the modified quasi-boundary value method to regularize the solution of problem (1) in the homogeneous backward case $f \equiv 0$. In particular, via the instability terms in the form of the solution of (1) ( $c f$. [4, Theorem 1.1]), they established an approximate problem by replacing $\mathcal{A}_{\alpha}=\mathcal{A}\left(I+\alpha \mathcal{A}^{-1}\right)$ for the operator $\mathcal{A}$ and taking the perturbation $\alpha$ into final conditions of the ill-posed problem, and obtained the convergence order $\alpha^{\theta}, \theta \in(0,1)$. Motivated by that work, this paper is devoted to investigating a new regularization method.

In the past, many approaches have been studied for solving ill-posed problems, especially the backward heat problems. For example, Lattès and Lions [14], Showalter [15] and Boussetila and Rebbani [16] used the quasi-reversibility method; in [17] Ames and Epperson applied the least squares method with Tikhonov-type regularization; Clark and Oppenheimer [18], Denche and Bessila [19] and Trong et al. [20] used the quasi-boundary value method. Moreover, some other methods that should be listed are the mollification method by Hao [21] and the operator-splitting method studied by Kirkup and Wadsworth [22]. To the best of the authors? knowledge, although there are many works on several types of parabolic backward problems, the theoretical literature on regularizing the inverse problems for ultraparabolic equations is very scarce. Therefore, proposing a regularization method for problem (1) is the scope of this paper.

Our work presented in this paper has the following features. At first, for ease of the reading, we summarize in Section 2 some well-known facts in a semi-group of operators and present the formula of the solution of (1). Secondly, in Section 3 we construct the regularized solution based on our method, then obtain the error estimate. Finally, a numerical example is given in Section 4 to illustrate the efficiency of the result.

\section{Preliminaries}

The operator $-\Delta$ is a positive self-adjoint unbounded linear operator on $L^{2}(0, \pi)$. Therefore, it can be applied to some elementary results in $[4,7,15,23-26]$. Particularly, the 
formula of the solution of problem (1) can be obtained by Lorenzi [4], and the authors in $[23,24]$ gave a detailed description on fundamental properties of the generalized operator. In this section, we thus recall those results which we want to apply to our main results in this paper. We list them and skip their proofs for conciseness.

In fact, we shall study in this section the generalized formula of the solution by the following operator equation in terms of semi-group theory.

$$
\begin{cases}u_{t}+u_{s}+\mathcal{A} u=f(t, s), & (t, s) \in[0, T] \times[0, T], \\ u(T, s)=\psi(s), & s \in[0, T], \\ u(t, T)=\varphi(t), & t \in[0, T],\end{cases}
$$

where $\mathcal{A}$ is a positive self-adjoint unbounded linear operator on the Hilbert space $\mathcal{H}$.

We denote by $\left\{E_{\lambda}, \lambda>0\right\}$ the spectral resolution of the identify associated to $\mathcal{A}$. Let us denote

$$
S(t)=e^{-t \mathcal{A}}=\int_{0}^{\infty} e^{-t \lambda} d E_{\lambda} \in \mathcal{L}(\mathcal{H}), \quad t \geq 0
$$

the $C_{0}$-semi-group of contractions generated by $-\mathcal{A}(\mathcal{L}(\mathcal{H})$ stands for the Banach algebra of bounded linear operators on $\mathcal{H}$ ). Then

$$
\mathcal{A} u=\int_{0}^{\infty} \lambda d E_{\lambda} u
$$

for all $u \in \mathcal{D}(\mathcal{A})$. In this connection, $u \in \mathcal{D}(\mathcal{A})$ iff the integral (3) exists, i.e.,

$$
\int_{0}^{\infty} \lambda^{2} d\left\|E_{\lambda} u\right\|^{2}<\infty
$$

For this family of operators $\{S(t)\}_{t \geq 0}$, we have:

1. $\|S(t)\| \leq 1$ for all $t \geq 0$;

2. the function $t \mapsto S(t), t>0$ is analytic;

3. for every real $r \geq 0$ and $t>0$, the operator $S(t) \in \mathcal{L}\left(\mathcal{H}, \mathcal{D}\left(\mathcal{A}^{r}\right)\right)$;

4. for every integer $k \geq 0$ and $t>0,\left\|S^{k}(t)\right\|=\left\|\mathcal{A}^{k} S(t)\right\| \leq c(k) t^{-k}$;

5. for every $x \in \mathcal{D}\left(\mathcal{A}^{r}\right), r \geq 0$, we have $S(t) \mathcal{A}^{r} x=\mathcal{A}^{r} S(t) x$.

Remark 1 In the sequel, let us denote

$$
\begin{aligned}
& D_{1}=\{(t, s) \in[0, T] \times[0, T]: 0 \leq s \leq t \leq T\} ; \\
& D_{2}=\{(t, s) \in[0, T] \times[0, T]: 0 \leq t \leq s \leq T\},
\end{aligned}
$$

and make some conditions on the given functions as follows:

(A1) $\varphi \in C([0, T]$; $\mathcal{D}(\mathcal{A})) \cap C^{1}([0, T] ; \mathcal{H})$;

(A2) $\psi \in C([0, T] ; \mathcal{D}(\mathcal{A})) \cap C^{1}([0, T] ; \mathcal{H})$;

(A3) $\varphi(0)=\psi(0)$;

(A4) $f \in C([0, T] \times[0, T] ; \mathcal{H}) \cap C^{1}\left(D_{1} \times D_{2} ; \mathcal{H}\right)$.

In the following theorems, we show the formula of the solution of problem (2) by employing Theorem 1.1 in [4] with $a_{1}(t)=a_{2}(s)=1$ and following the steps in [7]. 
Theorem 2 Under conditions (A1)-(A4), the problem

$$
\begin{cases}u_{t}+u_{s}+\mathcal{A} u=f(t, s), & (t, s) \in[0, T] \times[0, T] \\ u(0, s)=\psi(s), & s \in[0, T] \\ u(t, 0)=\varphi(t), & t \in[0, T]\end{cases}
$$

admits a unique solution $u$ presented by the following formula. For any $(t, s) \in D_{1}$,

$$
u(t, s)=S(s) \varphi(t-s)+\int_{0}^{s} S(s-\eta) f(t-s+\eta, \eta) d \eta
$$

and for any $(t, s) \in D_{2}$,

$$
u(t, s)=S(t) \psi(s-t)+\int_{0}^{t} S(t-\eta) f(\eta, s-t+\eta) d \eta .
$$

Moreover, the solution $u$ belongs to the space $C([0, T] \times[0, T] ; \mathcal{D}(\mathcal{A})) \cap C^{1}([0, T] \times$ $[0, T] ; \mathcal{H})$.

Theorem 3 Under conditions (A1)-(A4), if the problem

$$
\begin{cases}u_{t}+u_{s}-\mathcal{A} u=f(t, s), & (t, s) \in[0, T] \times[0, T] \\ u(0, s)=\psi(s), & s \in[0, T] \\ u(t, 0)=\varphi(t), & t \in[0, T]\end{cases}
$$

admits a solution $u$, then this solution can be presented by

$$
u(t, s)= \begin{cases}S^{-1}(t) \psi(s-t)+\int_{s-t}^{s} S(\eta-s) f(t-s+\eta, \eta) d \eta, & (t, s) \in D_{2}, \\ S^{-1}(s) \varphi(t-s)+\int_{t-s}^{t} S(\eta-t) f(\eta, \eta+s-t) d \eta, & (t, s) \in D_{1}\end{cases}
$$

Proof We put $\tau=T-t, \xi=T-s$ and write

$$
u(t, s)=u(T-\tau, T-\xi):=v(\tau, \xi)
$$

the function $v(\tau, \xi):[0, T] \times[0, T] \rightarrow \mathcal{H}$ satisfies problem (4), namely

$$
\begin{cases}v_{\tau}+v_{\xi}+\mathcal{A} v=F(\tau, \xi) \equiv-f(T-\tau, T-\xi), & (\tau, \xi) \in[0, T] \times[0, T], \\ v(0, \xi)=\psi_{1}(\xi) \equiv u(T, T-\xi), & \xi \in[0, T], \\ v(\tau, 0)=\varphi_{1}(\tau) \equiv u(T-\tau, T), & \tau \in[0, T] .\end{cases}
$$

Thanks to Theorem $2, v(\tau, \xi)$ is given by

$$
v(\tau, \xi)= \begin{cases}S(\xi) \varphi_{1}(\tau-\xi)+\int_{0}^{\xi} S(\xi-\eta) F(\tau-\xi+\eta, \eta) d \eta, & (\tau, \xi) \in D_{1}, \\ S(\tau) \psi_{1}(\xi-\tau)+\int_{0}^{\tau} S(\tau-\eta) F(\eta, \xi-\tau+\eta) d \eta, & (\tau, \xi) \in D_{2} .\end{cases}
$$


It follows that

$$
u(t, s)=\left\{\begin{array}{l}
S(T-s) u(T+t-s, T)-\int_{0}^{T-s} S(T-s-\eta) f(T+t-s-\eta, T-\eta) d \eta, \\
\quad(t, s) \in D_{2}, \\
S(T-t) u(T, T+s-t)-\int_{0}^{T-t} S(T-t-\eta) f(T-\eta, T+s-t-\eta) d \eta, \\
\quad(t, s) \in D_{1} .
\end{array}\right.
$$

Thus, we obtain

$$
u(t, s)= \begin{cases}S(T-s) u(T+t-s, T)-\int_{s}^{T} S(\zeta-s) f(t-s+\zeta, \zeta) d \zeta, & (t, s) \in D_{2}, \\ S(T-t) u(T, T+s-t)-\int_{t}^{T} S(\zeta-t) f(\zeta, \zeta+s-t) d \zeta, & (t, s) \in D_{1},\end{cases}
$$

by the maps $\zeta=T-\eta$ in the integrals. We can see by the initial conditions of (5) that

$$
\begin{aligned}
& u(t, 0)=\varphi(t)=S(T-t) u(T, T-t)-\int_{t}^{T} S(\zeta-t) f(\zeta, \zeta-t) d \zeta, \\
& u(0, s)=\psi(s)=S(T-s) u(T-s, T)-\int_{s}^{T} S(\zeta-s) f(\zeta-s, \zeta) d \zeta,
\end{aligned}
$$

which leads to

$$
\begin{cases}\varphi(t-s)=S(T-t+s) u(T, T-t+s)-\int_{t-s}^{T} S(\zeta-t+s) f(\zeta, \zeta-t+s) d \zeta, & (t, s) \in D_{1} \\ \psi(s-t)=S(T-s+t) u(T-s+t, T)-\int_{s-t}^{T} S(\zeta-s+t) f(\zeta-s+t, \zeta) d \zeta, & (t, s) \in D_{2}\end{cases}
$$

By virtue of semi-group properties, we get

$$
\left\{\begin{array}{l}
S^{-1}(s) \varphi(t-s)=S(T-t) u(T, T-t+s)-\int_{t-s}^{T} S(\zeta-t) f(\zeta, \zeta-t+s) d \zeta \\
\quad(t, s) \in D_{1}, \\
S^{-1}(t) \psi(s-t)=S(T-s) u(T-s+t, T)-\int_{s-t}^{T} S(\zeta-s) f(\zeta-s+t, \zeta) d \zeta \\
\quad(t, s) \in D_{2} .
\end{array}\right.
$$

Substituting (7) into (6), we thus have

$$
u(t, s)= \begin{cases}S^{-1}(t) \psi(s-t)+\int_{s-t}^{s} S(\zeta-s) f(t-s+\zeta, \zeta) d \zeta, & (t, s) \in D_{2}, \\ S^{-1}(s) \varphi(t-s)+\int_{t-s}^{t} S(\zeta-t) f(\zeta, \zeta+s-t) d \zeta, & (t, s) \in D_{1} .\end{cases}
$$

Theorem 4 Under conditions (A1), (A2) and (A4), ifproblem (2) with $\varphi(T)=\psi(T)$ admits a solution $u$, then this solution can be given by

$$
u(t, s)= \begin{cases}S^{-1}(T-t) \psi(T+s-t)+\int_{t}^{T} S(\eta-T) f(\eta-t, \eta-s) d \eta, & (t, s) \in D_{1}, \\ S^{-1}(T-s) \varphi(T+t-s)+\int_{s}^{T} S(\eta-T) f(\eta-t, \eta-s) d \eta, & (t, s) \in D_{2} .\end{cases}
$$

Proof Now we put $\tau=T-t$ and $\xi=T-s$, then write

$$
u(t, s)=u(T-\tau, T-\xi):=v(\tau, \xi),
$$


the function $v(\tau, \xi):[0, T] \times[0, T] \rightarrow \mathcal{H}$ satisfies problem (5), namely

$$
\begin{cases}v_{\tau}+v_{\xi}-\mathcal{A} v=F(\tau, \xi) \equiv-f(T-\tau, T-\xi), & (\tau, \xi) \in[0, T] \times[0, T], \\ v(0, \xi)=\psi_{1}(\xi) \equiv u(T, T-\xi), & \xi \in[0, T], \\ v(\tau, 0)=\varphi_{1}(\tau) \equiv u(T-\tau, T), & \tau \in[0, T] .\end{cases}
$$

Using Theorem 3, the solution $v(\tau, \xi)$ can be presented by

$$
v(\tau, \xi)= \begin{cases}S^{-1}(\tau) \psi_{1}(\xi-\tau)+\int_{\xi-\tau}^{\xi} S(\eta-\xi) F(\tau-\xi+\eta, \eta) d \eta, & (\tau, \xi) \in D_{2}, \\ S^{-1}(\xi) \varphi_{1}(\tau-\xi)+\int_{\tau-\xi}^{\tau} S(\eta-\tau) F(\eta, \eta+\xi-\tau) d \eta, & (\tau, \xi) \in D_{1}\end{cases}
$$

It follows that

$$
u(T-\tau, T-\xi)=\left\{\begin{array}{c}
S^{-1}(\tau) u(T, T-\xi+\tau)-\int_{\xi-\tau}^{\xi} S(\eta-\xi) f(T-\tau+\xi-\eta, T-\eta) d \eta, \\
(\tau, \xi) \in D_{2}, \\
S^{-1}(\xi) u(T-\tau+\xi, T)-\int_{\tau-\xi}^{\tau} S(\eta-\tau) f(T-\eta, T-\eta-\xi+\tau) d \eta, \\
(\tau, \xi) \in D_{1} .
\end{array}\right.
$$

Hence, we obtain

$$
\begin{aligned}
u(t, s)=\left\{\begin{array}{c}
S^{-1}(T-t) \psi(T+s-t)-\int_{t-s}^{T-s} S(\eta-T+s) f(T+t-s-\eta, T-\eta) d \eta, \\
(t, s) \in D_{1}, \\
S^{-1}(T-s) \varphi(T+t-s)-\int_{s-t}^{T-t} S(\eta-T+t) f(T-\eta, T+s-t-\eta) d \eta, \\
(t, s) \in D_{2},
\end{array}\right. \\
=\left\{\begin{array}{c}
S^{-1}(T-t) \psi(T+s-t)-\int_{t}^{T} S(\zeta-T) f(T+t-\zeta, T+s-\zeta) d \zeta, \\
(t, s) \in D_{1}, \\
S^{-1}(T-s) \varphi(T+t-s)-\int_{s}^{T} S(\zeta-T) f(T+t-\zeta, T+s-\zeta) d \zeta, \\
(t, s) \in D_{2},
\end{array}\right.
\end{aligned}
$$

which completes the proof.

Now we return to the consideration of problem (1). All of our results in this paper apply to more general problems, for which the boundary conditions are generalized in Robintype, for example,

$$
\begin{aligned}
& \alpha_{1} u(0, t, s)+\alpha_{2} u_{x}(0, t, s)=0, \\
& \alpha_{3} u(\pi, t, s)+\alpha_{4} u_{x}(\pi, t, s)=0,
\end{aligned}
$$

or we can consider, in general, the operator equations with the self-adjoint operator $\mathcal{A}$ having a discrete spectrum on an abstract Hilbert space $\mathcal{H}$ and satisfying the condition that $-\mathcal{A}$ generates a compact contraction semi-group on $\mathcal{H}$, like problem (2) considered above. However, for the sake of simplicity, we confine our attention to problem (1) in which the homogeneous Dirichlet boundary conditions at the endpoints of $[0, \pi]$ are given. In 
this problem, we have $\mathcal{H}=L^{2}(0, \pi)$ and $\mathcal{D}(\mathcal{A})=H_{0}^{1}(0, \pi) \cap H^{2}(0, \pi)$, so there exists an orthonormal basis of $L^{2}(0, \pi),\left\{\phi_{n}\right\}_{n \in \mathbb{N}}$ satisfying (see, e.g., [27, p.181])

$$
\phi_{n} \in H_{0}^{1}(0, \pi) \cap C^{\infty}([0, \pi]), \quad \Delta \phi_{n}=-\lambda_{n} \phi_{n}, \quad 0<\lambda_{1} \leq \lambda_{2} \leq \cdots \leq \lim _{n \rightarrow \infty} \lambda_{n}=\infty .
$$

The operator thus has a discrete spectrum $\sigma(\mathcal{A})=\left\{\lambda_{n}\right\}_{n \geq 1}$ with $\lambda_{n}=n^{2}$ and gives the orthonormal eigenbasis $\phi_{n}=\sqrt{\frac{2}{\pi}} \sin (n x)$ for $n \in \mathbb{N}, n \geq 1$. Then, thanks to those theorems above, the solution has the form

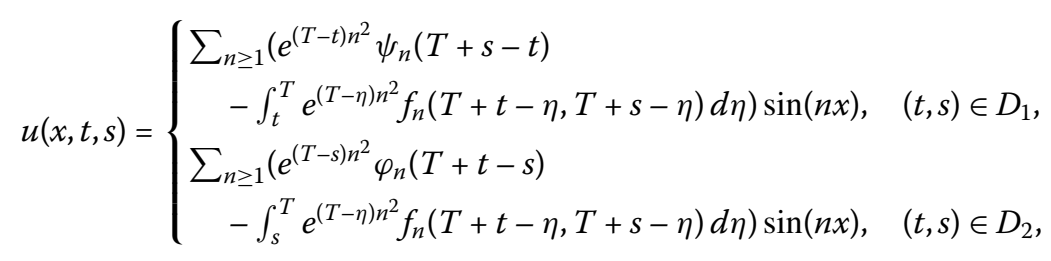

where

$$
\begin{aligned}
& \varphi_{n}(t)=\frac{2}{\pi} \int_{0}^{\pi} \varphi(x, t) \sin (n x) d x, \quad \psi_{n}(s)=\frac{2}{\pi} \int_{0}^{\pi} \psi(x, s) \sin (n x) d x, \\
& f_{n}(t, s)=\frac{2}{\pi} \int_{0}^{\pi} f(x, t, s) \sin (n x) d x .
\end{aligned}
$$

We can see that the instability is caused by all of the exponential functions. In fact, let us see the case $(t, s) \in D_{1}$ in (8). Since the discrete spectrum increases monotonically as $n$ tends to infinity, the rapid escalation of $e^{(T-t) n^{2}}$ and $e^{(T-\eta) n^{2}}$ is mainly the instability cause. Even though these exact given functions $\left(\psi_{n}, f_{n}\right)$ may tend to zero very fast, performing classical calculation is impossible. It is because the given data may be diffused by a variety of reasons such as round-off errors, measurement errors. A small perturbation in the data can arbitrarily generate a large error in the solution. A regularization method is thus required.

\section{Theoretical results}

In this section, assuming that the problem has an exact solution $u$ satisfying various corresponding assumptions, we construct the regularized solution depending continuously on the data such that it converges to the exact solution $u$ in some sense. Moreover, the accuracy of regularized solution is estimated.

The solution of (1) can be given by

$$
u(x, t, s)= \begin{cases}\sum_{n \geq 1} e^{(T-t) n^{2}}\left(\psi_{n}(T+s-t)\right. & \\ \left.\quad-\int_{t}^{T} e^{(t-\eta) n^{2}} f_{n}(T+t-\eta, T+s-\eta) d \eta\right) \sin (n x), & (t, s) \in D_{1}, \\ \sum_{n \geq 1} e^{(T-s) n^{2}}\left(\varphi_{n}(T+t-s)\right. & \\ \left.\quad-\int_{s}^{T} e^{(s-\eta) n^{2}} f_{n}(T+t-\eta, T+s-\eta) d \eta\right) \sin (n x), & (t, s) \in D_{2} .\end{cases}
$$

We shall replace all instability terms by the better ones, particularly $\left(\varepsilon+e^{-p n^{2}}\right)^{\frac{t-T}{p}}$ and $\left(\varepsilon+e^{-p n^{2}}\right)^{\frac{s-T}{p}}$, where $p \geq 1$ is a real number. Then the regularized solution corresponding 
to the exact data is

$$
\begin{aligned}
u^{\varepsilon}(x, t, s)= & \sum_{n \geq 1}\left(\varepsilon+e^{-p n^{2}}\right)^{\frac{t-T}{p}} \\
& \times\left(\psi_{n}(T+s-t)-\int_{t}^{T} e^{(t-\eta) n^{2}} f_{n}(T+t-\eta, T+s-\eta) d \eta\right) \sin (n x)
\end{aligned}
$$

for any $(t, s) \in D_{1}$, and

$$
\begin{aligned}
u^{\varepsilon}(x, t, s)= & \sum_{n \geq 1}\left(\varepsilon+e^{-p n^{2}}\right)^{\frac{s-T}{p}} \\
& \times\left(\varphi_{n}(T+t-s)-\int_{s}^{T} e^{(s-\eta) n^{2}} f_{n}(T+t-\eta, T+s-\eta) d \eta\right) \sin (n x)
\end{aligned}
$$

for any $(t, s) \in D_{2}$.

We also denote the regularized solution corresponding to the perturbed data by

$$
\begin{aligned}
v^{\varepsilon}(x, t, s)= & \sum_{n \geq 1}\left(\varepsilon+e^{-p n^{2}}\right)^{\frac{t-T}{p}} \\
& \times\left(\psi_{n}^{\varepsilon}(T+s-t)-\int_{t}^{T} e^{(t-\eta) n^{2}} f_{n}(T+t-\eta, T+s-\eta) d \eta\right) \sin (n x)
\end{aligned}
$$

for any $(t, s) \in D_{1}$, and

$$
\begin{aligned}
v^{\varepsilon}(x, t, s)= & \sum_{n \geq 1}\left(\varepsilon+e^{-p n^{2}}\right)^{\frac{s-T}{p}} \\
& \times\left(\varphi_{n}^{\varepsilon}(T+t-s)-\int_{s}^{T} e^{(s-\eta) n^{2}} f_{n}(T+t-\eta, T+s-\eta) d \eta\right) \sin (n x)
\end{aligned}
$$

for any $(t, s) \in D_{2}$.

Now we shall show two elementary inequalities in the following lemmas.

Lemma 5 For $0 \leq t \leq T \leq p$, we have

$$
\left(\varepsilon+e^{-n^{2} p}\right)^{\frac{t-T}{p}} \leq \varepsilon^{\frac{t-T}{p}} .
$$

Proof It is obvious that $\left(\varepsilon+e^{-n^{2} p}\right)^{\frac{t-T}{p}} \leq \varepsilon^{\frac{t-T}{p}}$ since $\varepsilon+e^{-n^{2} p} \geq \varepsilon$.

Lemma 6 For all $x>0,0<\alpha<1$, we have

$$
1-(x+1)^{-\alpha} \leq x^{1-\alpha} .
$$

Proof The proof of this lemma is based on the fact that $x^{\alpha}<(x+1)^{\alpha}$. Therefore, we have

$$
\begin{aligned}
1+x & \leq 1+x^{1-\alpha}(x+1)^{\alpha} \\
& \leq\left[1+x^{1-\alpha}(x+1)^{\alpha}\right]^{\frac{1}{\alpha}},
\end{aligned}
$$


which leads to

$$
\begin{aligned}
1-(x+1)^{-\alpha} & =\frac{(x+1)^{\alpha}-1}{(x+1)^{\alpha}} \\
& \leq \frac{1+x^{1-\alpha}(x+1)^{\alpha}-1}{(x+1)^{\alpha}} \\
& \leq x^{1-\alpha} .
\end{aligned}
$$

In the sequel, we only prove the case $(t, s) \in D_{1}$ in our main result because of the similarity. The results are about the regularized solution depending continuously on the corresponding data and the convergence of that solution to the exact solution. Now we shall use two elementary lemmas above to support the proof of the main results.

Lemma 7 Under conditions (A1), (A2), (A4) and assuming that $\varphi(T)=\psi(T)$, then the function $u^{\varepsilon}$ given by (10)-(11) depends continuously on $(\varphi, \psi)$ in $L^{2}(0, \pi)$.

Proof Let $u_{1}^{\varepsilon}$ and $u_{2}^{\varepsilon}$ be two solutions of (10)-(11) corresponding to the data $\left(\varphi^{1}, \psi^{1}\right)$ and $\left(\varphi^{2}, \psi^{2}\right)$, respectively. By using Parseval?s relation, for $\left(\{, s) \in D_{1}\right.$, we have

$$
\begin{aligned}
\left\|u_{1}^{\varepsilon}(\cdot, t, s)-u_{2}^{\varepsilon}(\cdot, t, s)\right\|^{2} & =\frac{\pi}{2} \sum_{n \geq 1}\left(\varepsilon+e^{-p n^{2}}\right)^{\frac{2(t-T)}{p}}\left(\psi_{n}^{1}(T+s-t)-\psi_{n}^{2}(T+s-t)\right)^{2} \\
& \leq \varepsilon^{\frac{2(t-T)}{p}}\left\|\psi^{1}(T+s-t)-\psi^{2}(T+s-t)\right\|^{2} .
\end{aligned}
$$

Similarly, for any $(t, s) \in D_{2}$, we get

$$
\begin{aligned}
\left\|u_{1}^{\varepsilon}(\cdot, t, s)-u_{2}^{\varepsilon}(\cdot, t, s)\right\|^{2} & =\frac{\pi}{2} \sum_{n \geq 1}\left(\varepsilon+e^{-p n^{2}}\right)^{\frac{2(s-T)}{p}}\left(\varphi_{n}^{1}(T+t-s)-\varphi_{n}^{2}(T+t-s)\right)^{2} \\
& \leq \varepsilon^{\frac{2(s-T)}{p}}\left\|\varphi^{1}(T+t-s)-\varphi^{2}(T+t-s)\right\|^{2} .
\end{aligned}
$$

Theorem 8 Under conditions (A1), (A2) and (A4), ifproblem (1) with $\varphi(T)=\psi(T)$ admits a unique solution $u$ satisfying

$$
\frac{\pi}{2} \sup _{(t, s) \in D_{1}} \sum_{n=1}^{\infty} e^{2(p+t-T) n^{2}}\left|u_{n}(t, s)\right|^{2}<C_{1}
$$

and

$$
\frac{\pi}{2} \sup _{(t, s) \in D_{2}} \sum_{n=1}^{\infty} e^{2(p+s-T) n^{2}}\left|u_{n}(t, s)\right|^{2}<C_{2},
$$

where $u_{n}(t, s)=\int_{0}^{\pi} u(x, t, s) \sin (n x) d x$, let $\left(\varphi^{\varepsilon}, \psi^{\varepsilon}\right)$ be perturbed functions satisfying conditions (A1)-(A2), respectively, and let $v^{\varepsilon}$ be the regularized solution, given by (12)-(13), corresponding to the perturbed data $\left(\varphi^{\varepsilon}, \psi^{\varepsilon}\right)$, then for $(t, s) \in D_{1}$ we have

$$
\left\|v^{\varepsilon}(\cdot, t, s)-u(\cdot, t, s)\right\| \leq\left(1+\sqrt{C_{1}}\right) \varepsilon^{\frac{t-T+p}{p}},
$$


and for $(t, s) \in D_{2}$,

$$
\left\|v^{\varepsilon}(\cdot, t, s)-u(\cdot, t, s)\right\| \leq\left(1+\sqrt{C_{2}}\right) \varepsilon^{\frac{s-T+p}{p}} .
$$

Proof For any $(t, s) \in D_{1}$, we have

$$
\begin{aligned}
u(x, t)=\sum_{n \geq 1} & e^{(T-t) n^{2}}\left(\psi_{n}(T+s-t)-\int_{t}^{T} e^{(t-\eta) n^{2}} f_{n}(T+t-\eta, T+s-\eta) d \eta\right) \sin (n x), \\
u^{\varepsilon}(x, t, s)= & \sum_{n \geq 1}\left(\varepsilon+e^{-p n^{2}}\right)^{\frac{t-T}{p}} \\
& \times\left(\psi_{n}(T+s-t)-\int_{t}^{T} e^{(t-\eta) n^{2}} f_{n}(T+t-\eta, T+s-\eta) d \eta\right) \sin (n x), \\
v^{\varepsilon}(x, t, s)= & \sum_{n \geq 1}\left(\varepsilon+e^{-p n^{2}}\right)^{\frac{t-T}{p}} \\
& \times\left(\psi_{n}^{\varepsilon}(T+s-t)-\int_{t}^{T} e^{(t-\eta) n^{2}} f_{n}(T+t-\eta, T+s-\eta) d \eta\right) \sin (n x) .
\end{aligned}
$$

Using the triangle inequality, in order to get the error estimate, we have to estimate $\left\|\nu^{\varepsilon}(\cdot, t, s)-u^{\varepsilon}(\cdot, t, s)\right\|$ and $\left\|u^{\varepsilon}(\cdot, t, s)-u(\cdot, t, s)\right\|$. Indeed, we get

$$
\begin{aligned}
\left\|v^{\varepsilon}(\cdot, t, s)-u^{\varepsilon}(\cdot, t, s)\right\|^{2} & =\frac{\pi}{2} \sum_{n \geq 1}\left(\varepsilon+e^{-p n^{2}}\right)^{\frac{2(t-T)}{p}}\left(\psi_{n}^{\varepsilon}(T+s-t)-\psi_{n}(T+s-t)\right)^{2} \\
& \leq \varepsilon^{\frac{2(t-T)}{p}}\left\|\psi^{\varepsilon}(T+s-t)-\psi(T+s-t)\right\|^{2} \\
& \leq \varepsilon^{\frac{2(t-T+p)}{p}} .
\end{aligned}
$$

Next, $\left\|u^{\varepsilon}(\cdot, t, s)-u(\cdot, t, s)\right\|$ can be estimated as follows. We put

$$
u_{n}(t, s)=e^{(T-t) n^{2}} \psi_{n}(T+s-t)-\int_{t}^{T} e^{(T-\eta) n^{2}} f_{n}(T+t-\eta, T+s-\eta) d \eta
$$

then we have

$$
\begin{aligned}
\left(1+\varepsilon e^{n^{2} p}\right)^{\frac{t-T}{p}} u_{n}(t, s)= & \left(\left(1+\varepsilon e^{n^{2} p}\right) e^{-p n^{2}}\right)^{\frac{t-T}{p}} \psi_{n}(T+s-t) \\
& -\int_{t}^{T}\left(1+\varepsilon e^{n^{2} p}\right)^{\frac{t-T}{p}} e^{(T-t) n^{2}} e^{(t-\eta) n^{2}} f_{n}(T+t-\eta, T+s-\eta) d \eta \\
= & \left(\varepsilon+e^{\left.-p n^{2}\right)^{\frac{t-T}{p}}} \psi_{n}(T+s-t)\right. \\
& -\int_{t}^{T}\left(\varepsilon+e^{-p n^{2}}\right)^{\frac{t-T}{p}} e^{(t-\eta) n^{2}} f_{n}(T+t-\eta, T+s-\eta) d \eta .
\end{aligned}
$$

Therefore, we conclude that

$$
\begin{aligned}
(\varepsilon & \left.+e^{-p n^{2}}\right)^{\frac{t-T}{p}}\left(\psi_{n}(T+s-t)-\int_{t}^{T} e^{(t-\eta) n^{2}} f_{n}(T+t-\eta, T+s-\eta) d \eta\right) \equiv u_{n}^{\varepsilon}(t, s) \\
& =\left(1+\varepsilon e^{n^{2} p}\right)^{\frac{t-T}{p}} u_{n}(t, s) .
\end{aligned}
$$


Now using Parseval?s relation again, we thus obtain

$$
\left\|u^{\varepsilon}(\cdot, t, s)-u(\cdot, t, s)\right\|^{2}=\frac{\pi}{2} \sum_{n \geq 1}\left|u_{n}^{\varepsilon}(t, s)-u_{n}(t, s)\right|^{2}=\frac{\pi}{2} \sum_{n \geq 1}\left(1-\left(1+\varepsilon e^{n^{2} p}\right)^{\frac{t-T}{p}}\right)^{2}\left|u_{n}(t, s)\right|^{2} .
$$

Thanks to Lemma 6 and assumption (14), we have

$$
\left\|u^{\varepsilon}(\cdot, t, s)-u(\cdot, t, s)\right\|^{2} \leq \frac{\pi}{2} \sum_{n \geq 1}\left(\varepsilon e^{n^{2} p}\right)^{2-\frac{2(T-t)}{p}}\left|u_{n}(t, s)\right|^{2} \leq \varepsilon^{2\left(1+\frac{t-T}{p}\right)} C_{1} .
$$

Combining (16)-(17), we obtain

$$
\begin{aligned}
\left\|v^{\varepsilon}(\cdot, t, s)-u(\cdot, t, s)\right\| & \leq\left\|v^{\varepsilon}(\cdot, t, s)-u^{\varepsilon}(\cdot, t, s)\right\|+\left\|u^{\varepsilon}(\cdot, t, s)-u(\cdot, t, s)\right\| \\
& \leq \varepsilon^{\frac{t-T+p}{p}}+\varepsilon^{1+\frac{t-T}{p}} \sqrt{C_{1}} \leq\left(1+\sqrt{C_{1}}\right) \varepsilon^{\frac{t-T+p}{p}} .
\end{aligned}
$$

Similarly, we obtain the error estimate

$$
\left\|v^{\varepsilon}(\cdot, t, s)-u(\cdot, t, s)\right\| \leq\left(1+\sqrt{C_{2}}\right) \varepsilon^{\frac{s-T+p}{p}}
$$

for the case $(t, s) \in D_{2}$ with assumption (15).

Hence, we complete the proof.

Remark 9 From Theorem 8 , we can see that $v^{\varepsilon}(\cdot, t, s)$ strongly converges to $u(\cdot, t, s)$ in $L^{2}(0, \pi)$ for any $(t, s) \in[0, T] \times[0, T]$ as $\varepsilon$ tends to zero. One advantage of this method is that the endpoints of time $[0, T] \times[0, T]$, for example, $(t, s)=(0,0)$ and $(t, s)=(T, T)$, nearly have the same rate of convergence in some cases. Indeed, the convergence speed at $(t, s)=(0,0)$ is $\varepsilon^{\frac{p-T}{p}}$ and it is of order $\varepsilon$ for $(t, s)=(T, T)$. Then, if $p$ is very large for any fixed $T>0$, the order $\varepsilon^{\frac{p-T}{p}}$ may approach $\varepsilon$. This creates the globally stable behavior of the error in numerical sense. On the other hand, the natural acceptance of (14)-(15) can be obtained at $(t, s)=(0,0)$. Namely, by letting $p=T$ the conditions become

$$
\frac{\pi}{2} \sum_{n=1}^{\infty}\left|u_{n}(0,0)\right|^{2}=\|u(\cdot, 0,0)\|^{2}
$$

Moreover, the error is of order $\mathcal{O}\left(\varepsilon^{\frac{p-T}{p}}\right)$ for all $(t, s) \in[0, T] \times[0, T]$. If $p>T$, this error is faster than the order $\ln \left(\varepsilon^{-1}\right)^{-q}, q>0$ as $\varepsilon \rightarrow 0$ which is studied in many works, such as $[18-20,23]$. Combining the strong points above, the reader can infer that our method is feasible.

\section{A numerical example}

In order to see how well the method works, we consider problem (1) by choosing

$$
f(x, t, s)=-2 e^{-2 t-s} \sin x, \quad \psi(x, s)=e^{-2-s} \sin x, \quad \varphi(x, t)=e^{-2 t-1} \sin x,
$$

and the domain $[0, \pi] \times[0,1]^{2}$. For those given functions, the problem has a unique solution

$$
u_{e x}(x, t, s)=e^{-2 t-s} \sin x
$$




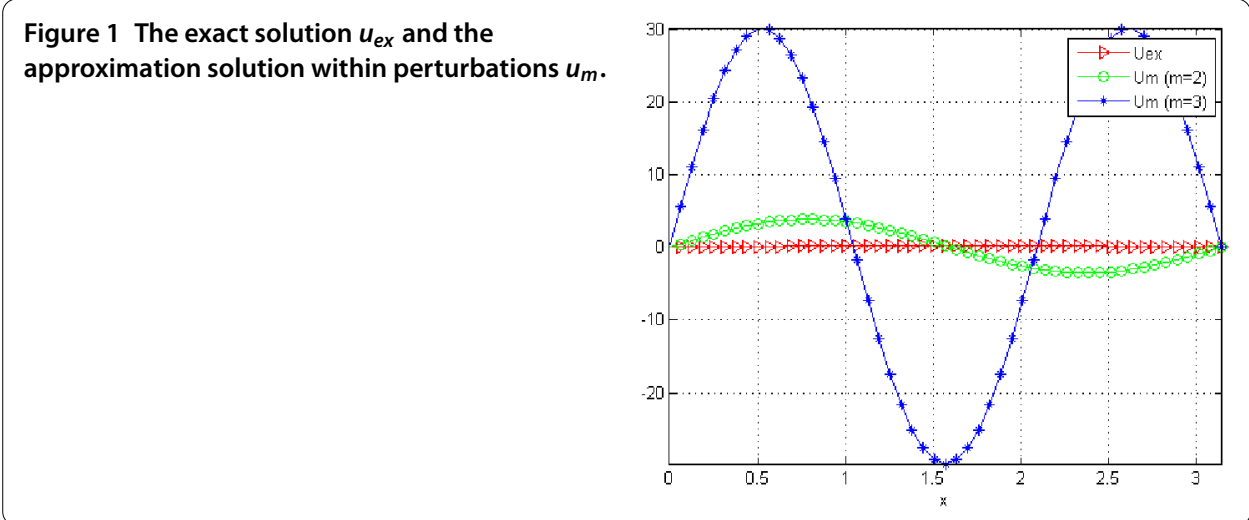

Now let us take perturbation on data functions as follows. For $m \in \mathbb{N}$, we define

$$
\begin{aligned}
& \varphi_{m}(x, t)=e^{-2 t-1} \sin x+\frac{\sin (m x)}{m}, \\
& \psi_{m}(x, s)=e^{-s-2} \sin x+\frac{\sin (m x)}{m} .
\end{aligned}
$$

Thus, the solution corresponding to the perturbed data functions is

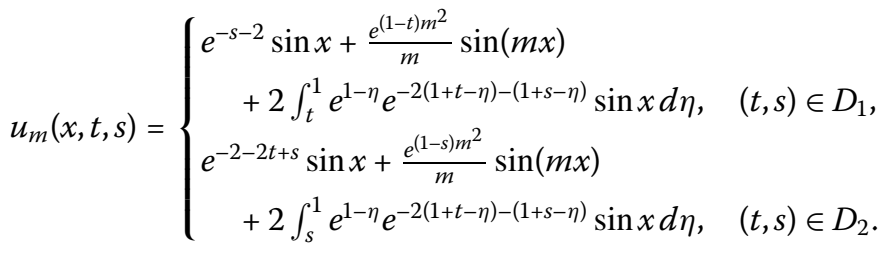

$$
\begin{aligned}
& = \begin{cases}e^{-s-2} \sin x+\frac{e^{(1-t) m^{2}}}{m} \sin (m x)+e^{-2 t-s-2}\left(e^{2}-e^{2 t}\right) \sin x, & (t, s) \in D_{1}, \\
e^{-2-2 t+s} \sin x+\frac{e^{(1-s) m^{2}}}{m} \sin (m x)+e^{-2 t-s-2}\left(e^{2}-e^{2 s}\right) \sin x, & (t, s) \in D_{2} .\end{cases}
\end{aligned}
$$

It is easy to see that $\left(\varphi_{m}, \psi_{m}\right)$ converges to $(\varphi, \psi)$ over the norm $L^{2}(0, \pi)$ as $m \rightarrow \infty$. To observe the ill-posedness, we can compute, for example, $u_{e x}\left(x, \frac{1}{2}, \frac{1}{2}\right)=e^{-\frac{3}{2}} \sin x$ and

$$
u_{m}\left(x, \frac{1}{2}, \frac{1}{2}\right)=e^{\frac{-3}{2}} \sin x+\frac{e^{\frac{m^{2}}{2}}}{m} \sin (m x) \text {. }
$$

Therefore, we get

$$
\left\|u_{m}\left(\cdot, \frac{1}{2}, \frac{1}{2}\right)-u\left(\cdot, \frac{1}{2}, \frac{1}{2}\right)\right\|^{2}=\int_{0}^{\pi} \frac{e^{m^{2}}}{m^{2}} \sin ^{2}(m x) d x=\frac{\pi e^{m^{2}}}{2 m^{2}} \rightarrow \infty
$$

as $m \rightarrow \infty$. This divergence is also shown in Figure 1 with $m=2$ and $m=3$.

Now we compute the regularized solution based on (12)-(13) as follows.

$$
\begin{aligned}
v_{m}(x, t, s)= & \left(\sqrt{\frac{\pi}{2}} \frac{1}{m}+e^{-p}\right)^{\frac{t-1}{p}} e^{-3-s+t} \sin x+\left(\sqrt{\frac{\pi}{2}} \frac{1}{m}+e^{-p m^{2}}\right)^{\frac{t-1}{p}} \frac{\sin (m x)}{m} \\
& +2\left(\sqrt{\frac{\pi}{2}} \frac{1}{m}+e^{-p}\right)^{\frac{t-1}{p}} \int_{t}^{1} e^{t-\eta} e^{-2(1+t-\eta)-(1+s-\eta)} \sin x d \eta
\end{aligned}
$$




$$
\begin{aligned}
= & \left(\sqrt{\frac{\pi}{2}} \frac{1}{m}+e^{-p}\right)^{\frac{t-1}{p}}\left(e^{-3-s+t}+e^{-3-t-s}\left(e^{2}-e^{2 t}\right)\right) \sin x \\
& +\left(\sqrt{\frac{\pi}{2}} \frac{1}{m}+e^{-p m^{2}}\right)^{\frac{t-1}{p}} \frac{\sin (m x)}{m} \\
= & \left(\sqrt{\frac{\pi}{2}} \frac{1}{m}+e^{-p}\right)^{\frac{t-1}{p}} e^{-1-t-s} \sin x+\left(\sqrt{\frac{\pi}{2}} \frac{1}{m}+e^{-p m^{2}}\right)^{\frac{t-1}{p}} \frac{\sin (m x)}{m},
\end{aligned}
$$

for any $(t, s) \in D_{1}$, and

$$
\begin{aligned}
v_{m}(x, t, s)= & \left(\sqrt{\frac{\pi}{2}} \frac{1}{m}+e^{-p}\right)^{\frac{s-1}{p}} e^{-3-2 t+2 s} \sin x+\left(\sqrt{\frac{\pi}{2}} \frac{1}{m}+e^{-p m^{2}}\right)^{\frac{s-1}{p}} \frac{\sin (m x)}{m}, \\
& +2\left(\sqrt{\frac{\pi}{2}} \frac{1}{m}+e^{-p}\right)^{\frac{s-1}{p}} \int_{s}^{1} e^{s-\eta} e^{-2(1+t-\eta)-(1+s-\eta)} \sin x d \eta \\
= & \left(\sqrt{\frac{\pi}{2}} \frac{1}{m}+e^{-p}\right)^{\frac{s-1}{p}}\left(e^{-3-2 t+2 s}+e^{-3-2 t}\left(e^{2}-e^{2 s}\right)\right) \sin x \\
& +\left(\sqrt{\frac{\pi}{2}} \frac{1}{m}+e^{-p m^{2}}\right)^{\frac{s-1}{p}} \frac{\sin (m x)}{m} \\
= & \left(\sqrt{\frac{\pi}{2}} \frac{1}{m}+e^{-p}\right)^{\frac{s-1}{p}} e^{-1-2 t} \sin x+\left(\sqrt{\frac{\pi}{2}} \frac{1}{m}+e^{-p m^{2}}\right)^{\frac{s-1}{p}} \frac{\sin (m x)}{m}
\end{aligned}
$$

for any $(t, s) \in D_{2}$.

To obtain numerical results, we use a uniform grid of mesh-points $(x, t, s)=\left(x_{j}, t_{k}, s_{m}\right)$, where

$$
\begin{aligned}
& x_{j}=j \Delta x, \quad \Delta x=\frac{\pi}{K}, j=\overline{0, K}, \\
& t_{k}=k \Delta t, \quad s_{l}=l \Delta s, \quad \Delta t=\Delta s=\frac{1}{M}, k, l=\overline{0, M} .
\end{aligned}
$$

We thus seek the discrete solutions $u_{e x}^{j, k, l}=u_{e x}\left(x_{j}, t_{k}, s_{l}\right)$ and $v_{m}^{j, k, l}=v_{m}\left(x_{j}, t_{k}, s_{l}\right)$ given by (18) and (19)-(20), respectively.

By fixing $K=100, M=80$ and $p=10$, the numerical results are shown in Table 1 and illustrated in Figures 2 and 3. Figure 2 is the graphical representations for curved surfaces of the exact solution $(t, s) \mapsto u_{e x}\left(\frac{\pi}{2}, t, s\right) \equiv e^{-2 t-s}$ and of the approximate solution $(t, s) \mapsto$ $v_{m}\left(\frac{\pi}{2}, t, s\right)$ determined in (19)-(20) with $m=10^{10}$. In Figure 3, we have drawn the exact

Table 1 Comparison of absolute errors between the regularized solutions $v_{m}$ of $m=10^{2}$ and $m=10^{10}$

\begin{tabular}{llllll}
\hline $\boldsymbol{( x , \boldsymbol { t } , \boldsymbol { s } )}$ & Exact value & $\begin{array}{l}\text { App. value } \mathbf{1} \\
\left(\boldsymbol{m}=\mathbf{1 0}^{\mathbf{2}}\right)\end{array}$ & $\begin{array}{l}\text { App. value 2 } \\
\left(\boldsymbol{m}=\mathbf{1 0}^{\mathbf{1 0}}\right)\end{array}$ & Abs. error 1 & Abs. error 2 \\
\hline$\left(\frac{\pi}{2}, 0.75,0.75\right)$ & 0.1053992246 & 0.0915741799 & 0.1053992172 & 0.0138250446 & $7.4 \mathrm{E}-09$ \\
$\left(\frac{\pi}{2}, 0.5,0.5\right)$ & 0.2231301601 & 0.1684339068 & 0.2231301293 & 0.0546962533 & $3.08 \mathrm{E}-08$ \\
$\left(\frac{\pi}{2}, 0.25,0.25\right)$ & 0.4723665527 & 0.3098032761 & 0.4723664549 & 0.1625632766 & $9.87 \mathrm{E}-08$ \\
$\left(\frac{\pi}{2}, 0.125,0.125\right)$ & 0.6872892788 & 0.4201595585 & 0.6872891127 & 0.2671297203 & $1.661 \mathrm{E}-07$ \\
$\left(\frac{\pi}{2}, 0,0\right)$ & 1 & 0.5698263001 & 0.9999997239 & 0.4301736999 & $2.761 \mathrm{E}-07$ \\
\hline
\end{tabular}


Figure 2 Plot of the exact and regularized solutions at the midpoint of $[0, \pi]$.

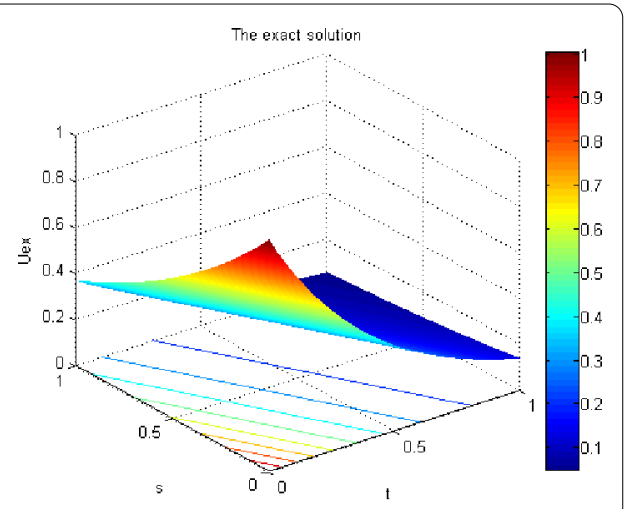

(a) Exact

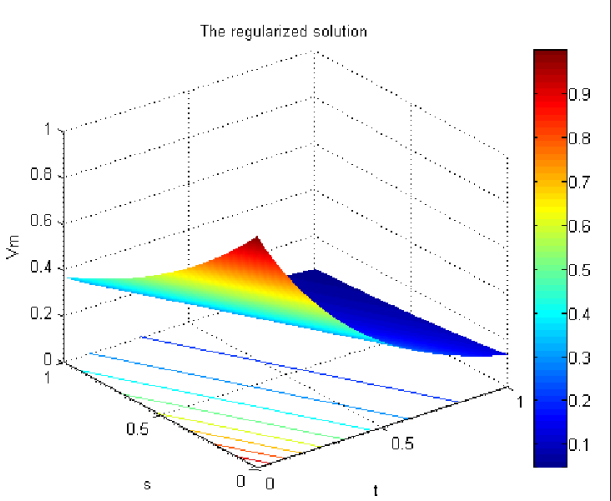

(b) Regularized $\left(m=10^{10}\right)$
Figure 3 Plot of absolute errors at the endpoint of time $(t, s)=(0,0)$.

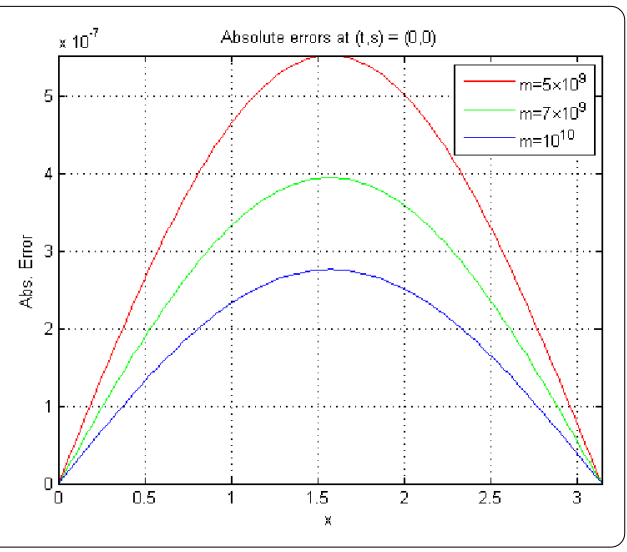

solution $x \mapsto u_{e x}(x, 0,0) \equiv \sin x$ and the approximate solution $x \mapsto v_{m}(x, 0,0)$, where $m$ are $5 \times 10^{9}, 7 \times 10^{9}$ and $10^{10}$, respectively, in order to see the convergence at $(t, s)=(0,0)$ as $m$ becomes very large, namely the bound $\varepsilon$ in theoretical result tends to zero. As in Figures 2 and 3, we can conclude that the regularized solution converges to the exact one as the error becomes smaller and smaller. Moreover, convergence is, particularly, observed from the absolute (abs.) errors in Table 1. Hence, our numerical results are all reasonable for the theoretical result. 


\section{Conclusion}

In this work, a regularization method has been successfully applied to the inverse ultraparabolic problem. This method is to replace the instability terms appearing in the formula of the solution which is employed by semi-group theory. Therefore, such a way forms the so-called regularized solution which strongly converges to the exact solution in $L^{2}$-norm. We also obtain the error estimate which is of order $\varepsilon^{\frac{p-T}{p}}, p>T$. By a numerical example, application of the method is flexible and calculation of successive approximations is direct and straightforward. This work is more general than [7], a recent work of Zouyed and Rebbani, in both error estimate and the considered problem.

\section{Competing interests}

The authors declare that they have no competing interests

\section{Authors? contributions}

VAK, LTL organized and wrote this manuscript. VAK, LTL and TTH contributed to all the steps of the proofs in this research together. NHT participated in the discussion and corrected the main results. All authors read and approved the final manuscript.

\section{Author details}

${ }^{1}$ Applied Analysis Research Group, Faculty of Mathematics and Statistics, Ton Duc Thang University, Ho Chi Minh City, Vietnam. ${ }^{2}$ Mathematics and Computer Science Division, Gran Sasso Science Institute, Viale Francesco Crispi 7, L?Aquila, Abruzzo 67100, Italy. ${ }^{3}$ Department of Mathematics and Computer Science, Ho Chi Minh City University of Science, 227 Nguyen Van Cu Street, District 5, Ho Chi Minh City, Vietnam.

\section{Acknowledgements}

The authors wish to express their sincere thanks to the anonymous referees and the handling editor for many constructive comments leading to the improved version of this paper.

Received: 20 September 2014 Accepted: 13 December 2014 Published online: 14 January 2015

\section{References}

1. Kozhanov, Al: On the solvability of boundary value problems for quasilinear ultraparabolic equations in some mathematical models of the dynamics of biological systems. J. Appl. Ind. Math. 4(4), 512-525 (2010)

2. Uhlenbeck, GE, Ornstein, LS: On the theory of the Brownian motion. Phys. Rev. 36, 823-841 (1930)

3. Chandrasekhar, S: Stochastic problems in physics and astronomy. Rev. Mod. Phys. 15, 1-89 (1943). Reprinted in Selected Papers On Noise And Stochastic Processes (Ed. N Wax). Dover, New York (1954)

4. Lorenzi, L: An ultraparabolic integrodifferential equation. Matematiche 58(2), 401-435 (1998)

5. Akrivis, G, Crouzeix, M, Thomée, V: Numerical methods for ultraparabolic equations. Calcolo 31(3-4), 179-190 (1994)

6. Genčev, TG: Ultraparabolic equations. Dokl. Akad. Nauk SSSR 151, 265-268 (1963). English Transl.

7. Zouyed, F, Rebbani, F: A modified quasi-boundary value method for an ultraparabolic ill-posed problem. J. Inverse III-Posed Probl. 22(4), 449-466 (2014)

8. Francesco, MD, Pascucci, A: A continuous dependence result for ultraparabolic equations in option pricing. J. Math. Anal. Appl. 336, 1026-1041 (2007)

9. Bensoussan, A, Chow, PL, Lions, JL: Filtering theory for stochastic process with two-dimensional time parameter. Math. Comput. Simul. 22(3), 213-221 (1980)

10. Tersenov, SA: Well-posedness of boundary value problems for a certain ultraparabolic equation. Sib. Math. J. 40, 6 (1999)

11. Dron?, VS, Ivasyshen, SD: Properties of the fundamental solutions and uniqueness theorems for the solutions of the Cauchy problem for one class of ultraparabolic equations. Ukr. Math. J. 50, 11 (1998)

12. Ashyralyev, A, Yilmaz, S: An approximation of ultra-parabolic equations. Abstr. Appl. Anal. 2012, Article ID 840621 (2012)

13. Marcozzi, MD: Extrapolation discontinuous Galerkin method for ultraparabolic equations. J. Comput. Appl. Math. 224, 679-687 (2009)

14. Lattès, R, Lions, JL: The Method of Quasi-Reversibility. Applications to Partial Differential Equations. Elsevier, New York (1969)

15. Showalter, RE: The final value problem for evolution equations. J. Math. Anal. Appl. 47, 563-572 (1974)

16. Boussetila, N, Rebbani, F: A modified quasi-reversibility method for a class of ill-posed Cauchy problems. Georgian Math. J. 14(4), 627-642 (2007)

17. Ames, KA, Epperson, JF: A kernel-based method for the approximate solutions of backward parabolic problems. SIAM J. Numer. Anal. 34, 1357-1390 (1997)

18. Clark, GW, Oppenheimer, SF: Quasireversibility methods for non-well-posed problems. Electron. J. Differ. Equ. 1994, 08 (1994)

19. Denche, M, Bessila, K: A modified quasi-boundary value method for ill-posed problems. J. Math. Anal. Appl. 301 419-426 (2005)

20. Trong, DD, Quan, PH, Khanh, TV, Tuan, NH: A nonlinear case of the 1-D backward heat problem: regularization and error estimate. Z. Anal. Anwend. 26(2), 231-245 (2007) 
21. Hao, DN: A mollification method for ill-posed problems. Numer. Math. 68, 469-506 (1994)

22. Kirkup, SM, Wadsworth, M: Solution of inverse diffusion problems by operator-splitting methods. Appl. Math. Model. 26, 1003-1018 (2002)

23. Tuan, NH, Trong, DD, Quan, PH: On a backward Cauchy problem associated with continuous spectrum operator. Nonlinear Anal. 73, 1966-1972 (2010)

24. Pazy, A: Semigroups of Linear Operators and Applications to Partial Differential Equations. Appl. Math. Sci., vol. 44. Springer, New York (1983)

25. Lunardi, A: Analytic Semigroups and Optimal Regularity in Parabolic Problems. Birkhäuser, Basel (1995)

26. Showalter, RE: Hilbert space methods for partial differential equations. Electron. J. Differ. Equ., Monograph 01 (1994)

27. Kwiecińska, AA: Stabilization of partial differential equations by noise. Stoch. Process. Appl. 79, 179-184 (1999)

Submit your manuscript to a SpringerOpen ${ }^{\circ}$ journal and benefit from:

- Convenient online submission

- Rigorous peer review

- Immediate publication on acceptance

- Open access: articles freely available online

- High visibility within the field

- Retaining the copyright to your article 